\title{
Correction to: Associations of recognition at work with subsequent health and quality of life among older working adults
}

\author{
Dorota Weziak-Bialowolska $^{1,2}$ (D) $\cdot$ Piotr Bialowolski $i^{1,2,3}$ (D)
}

Published online: 3 February 2022

○) Springer-Verlag GmbH Germany, part of Springer Nature 2022

\section{Correction to: \\ International Archives of Occupational and Environmental Health \\ https://doi.org/10.1007/s00420-021-01804-w}

In the original publication of the article the second author's affilaition was incorrectly published.

The correct affilation of Piotr Bialowolski is as follows:

Piotr Bialowolski ${ }^{1,2,3}$

https://orcid.org/0000-0003-4102-0107

${ }^{1}$ Sustainability and Health Initiative (SHINE), Department of Environmental Health, Harvard T. H. Chan School of Public Health; 665 Huntington Avenue, Boston, MA., 02115, USA
The original article can be found online at https://doi.org/10.1007/ s00420-021-01804-w.

Dorota Weziak-Bialowolska

doweziak@hsph.harvard.edu

1 Sustainability and Health Initiative (SHINE), Department of Environmental Health, Harvard T. H. Chan School of Public Health, 665 Huntington Avenue, Boston, MA 02115, USA

2 Human Flourishing Program, Institute for Quantitative Social Science, Harvard University, 129 Mount Auburn Street, Cambridge, MA 02138, USA

3 Department of Economics, Kozminski University, Jagiellońska 57, 03301 Warsaw, Poland
${ }^{2}$ Human Flourishing Program, Institute for Quantitative Social Science, Harvard University, 129 Mount Auburn Street, Cambridge, MA, 02138, USA

${ }^{3}$ Department of Economics, Kozminski University, Jagiellońska 57, 03 301, Warsaw, Poland

This has been corrected in this paper.

Publisher's Note Springer Nature remains neutral with regard to jurisdictional claims in published maps and institutional affiliations. 